\title{
Produção de cebola em solo salinizado ${ }^{1}$
}

\author{
$\overline{\text { Maria D. B. Lima² \& Leonardo T. Bull }}{ }^{3}$
}

\begin{abstract}
RESUMO
O uso de águas ou solos com elevada concentração de sais é uma das principais causas de insucesso na produção de muitas culturas razão por que se objetivou, com este trabalho, avaliar os efeitos de diferentes níveis de salinidade e de umidade do solo sobre a produção da cebola (cultivar Baia periforme). Para isto, conduziu-se um experimento em casa de vegetação do Departamento de Ciências do Solo da Faculdade de Ciências Agronômicas da UNESP - Botucatu, SP, em vasos de $10 \mathrm{~L}$. O solo foi salinizado com $\mathrm{NaCl}$, a fim de elevar o nível de condutividade elétrica do extrato de saturação do solo, de 0,13 para 2; 4; 6 e $8 \mathrm{dS} \mathrm{m}^{-1}$. A cada unidade experimental foram incorporados 15,2 g de termofosfato enriquecido com micronutrientes e, ainda, 1,5 L de matéria orgânica. Os tratamentos de umidade consistiram em se manter o solo nas faixas de 25-50, 50-75 e 75-100\% da capacidade de campo. Avaliaram-se altura da planta, diâmetro do pseudocaule e a produção de bulbos. Pelos resultados, concluiu-se que a salinidade do solo inibiu o desenvolvimento vegetativo e a produção de bulbos da cebola. A umidade do solo influenciou o diâmetro e o peso dos bulbos. O consumo de água pelas plantas diminuiu com a elevação da concentração de sais no solo.
\end{abstract}

Palavras-chave: Allium cepa, salinidade, água no solo

\section{Onion production in salinized soil}

\begin{abstract}
The use of water or soil with a high salt concentration has been the main cause of failure in the yield of many crops. Consequently, this work aimed at evaluating the effects of different salinity levels and soil moisture on onion yield (cultivar Baia periforme). An experiment was conducted in a greenhouse of the Soil Science Department - FCA - UNESP, Botucatu, $\mathrm{SP}$, in pots of $10 \mathrm{~L}$. The soil was salinized with $\mathrm{NaCl}$ in order to increase the electrical condutivity of saturation extract of soil from 0.13 to $2 ; 4 ; 6$ and $8 \mathrm{dS} \mathrm{m}^{-1}$. In each experimental unit $15.2 \mathrm{~g}$ of thermophosphate enriched by micronutrients and $1.5 \mathrm{~L}$ of organic matter were incorporated. The moisture treatments kept the soil within the limits of 25-50, 50-75 and $75-100 \%$ of field capacity. The plant height the pseudostem diameter and yield of bulbs were evaluated. The results lead to the conclusion that the salinity reduced the vegetative growth and the yield of onion bulbs. Moisture treatment influenced both the diameter and weight of bulbs. The water consumption of the plants decreased with increassing soil salt concentration.
\end{abstract}

Key words: Allium cepa, salinity, water stress 


\section{INTRODUÇÃO}

O aumento da concentração de sais em algumas áreas irrigadas é um problema de ordem mundial. Milhões de hectares de terra em todo o mundo são muito salinos e a cada ano se tornam mais improdutivas, devido à acumulação de sais presentes nas águas de irrigação. O uso de água ou solos com altos níveis de sais é uma das principais causas de insucesso na produção das culturas.

A cebola, Allium cepa L., preferida por suas características condimentares e propriedades terapêuticas, é uma das olerícolas mais significativas, do ponto de vista de volume de consumo e valor econômico.

No Nordeste brasileiro o cultivo da cebola representa expressiva fonte de renda para os agricultores das áreas irrigadas do Médio São Francisco. O impacto da salinidade do solo sobre a produtividade agrícola se torna fator limitante nessas áreas, que dependem da irrigação para um suprimento adequado de água às culturas. Os problemas de salinização desses solos contribuem para a redução acentuada do crescimento e produtividade de culturas desenvolvidas nessas regiões.

A sensibilidade às condições de estresse salino é variável de acordo com a espécie e a fase de germinação de cada cultura. Dias et al. (2003) consideram a germinação e o desenvolvimento inicial, as fases mais sensíveis aos efeitos da salinidade, porém Millar (1984) define como período mais crítico o início da formação dos bulbos de cebola. Wannamaker \& Pike (1987) não encontraram diferença significativa nas taxas de germinação entre cultivar de cebola ou níveis de sais nos tratamentos que receberam 0,0 a 25,0 dS m-1.

De acordo com Lima (1997), a redução na absorção de água, a toxicidade de íons específicos e os efeitos indiretos dos sais nos processos fisiológicos, são fatores responsáveis pela redução do crescimento das plantas. Segundo Ayers \& Westcot (1999), o rendimento das culturas cai significantemente quando o teor de sais na solução do solo prejudica a absorção de água e nutrientes pelas culturas, resultando em perdas no crescimento, desenvolvimento e produção (Lacerda, 2005; Gheyi et al., 2005). Em geral, esses decréscimos estão associados à redução na capacidade fotossintética devido ao declínio na condutância estomatal (Ziska et al., 1990; Sousa, 2006).

O trabalho teve por objetivo avaliar o rendimento da cultura de cebola, níveis de salinidade, em solo com diferentes, faixas de umidade.

\section{MATERIAL E MÉTODOS}

O experimento foi conduzido em casa de vegetação do Departamento de Ciências do Solo, da Faculdade de Ciências Agronômicas da UNESP - "Campus” de Botucatu, SP; o solo utilizado é classificado como Neossolo Flúvico distrófico (EMBRAPA, 1999), coletado na camada superficial $(0-30 \mathrm{~cm})$, em área pertencente à Faculdade de Ciências Agronômicas UNESP - Campus de Botucatu, SP; as análises químicas e da textura do solo são apresentadas na Tabela 1.

As mudas da cebola (Allium cepa), cultivar Baia periforme, foram transplantadas aos 75 dias após a semeadura; esta variedade apresenta, como características, bulbo de formato periforme, pescoço fino, película de cor amarela e tolera longo período de armazenamento (Mascarenhas, 1980).

O volume de água foi aplicado com base na pesagem diária dos vasos, a fim de repor a água evapotranspirada de cada tratamento predeterminada em função da água disponível (capacidade de campo - ponto de murcha permanente) e da curva característica de umidade do solo, observando-se a faixa de umidade para cada tratamento. Por outro lado, o peso das plantas nos vasos foi estimado em função da pesagem de plantas de cebola (indicadoras) conduzidas ao lado e nas mesmas condições ambientais das demais, para avaliar o peso da planta no decorrer do experimento, em função do seu crescimento.

O experimento consistiu de delineamento inteiramente casualizado, em um fatorial $5 \times 3$, correspondendo aos níveis de salinidade (5) e faixas de umidade do solo (3), e foi instalado em quarenta e cinco vasos plásticos com capacidade para $10 \mathrm{~L}$ recebendo, cada vaso, 8,0 kg de solo seco passado em peneira com malha de $4 \mathrm{~mm}$, adubado com 15,2 $\mathrm{g}$ de termofosfato enriquecido com micronutrientes (Yoorin Master) e ainda 1,5 L de matéria orgânica.

A cada tratamento foram acrescentados 2,50, 9,57, 16,67 e 23,70 g de $\mathrm{NaCl}$ para a salinização; as respectivas doses do sal foram determinadas mediante teste realizado previamente, visando elevar a condutividade elétrica do solo inicial, de 0,13 (testemunha), para valores de condutividade elétrica do

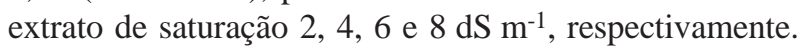

Após a salinização os vasos foram irrigados com o propósito de atingir a capacidade de campo do solo; em seguida, foram cobertos com plástico e deixados em incubação durante duas semanas, procedendo-se depois ao transplantio das mudas de cebola; semanalmente, foram avaliados o diâmetro do pseudocaule e a altura da planta medindo-se o comprimento, desde o solo até a extremidade da maior folha; enfim, para a análise estatística considerou-se apenas a última mensuração, aos 97 dias após o transplante.

A colheita das plantas (iniciada aos 98 dias após o transplantio) se deu em datas distintas, em função da maturação (estalo) em períodos diferentes obtendo-se, nesta ocasião, o peso total das plantas (ramas + bulbos); após o período de cura, realizado à sombra e em temperatura ambiente, procedeu-se à nova pesagem, separando-se o bulbo da parte aérea de cada planta.

A eficiência do uso da água foi definida pela relação entre a produção de matéria seca (g) da parte aérea por vaso e

Tabela 1. Atributos químicos e granulometria do solo utilizado no experimento

\begin{tabular}{|c|c|c|c|c|c|c|c|c|c|c|c|c|}
\hline pH em & M.0. & $P$ & $\mathrm{H}^{+}+\mathrm{Al}^{3+}$ & $\mathrm{K}^{+}$ & $\mathrm{Ca}^{2+}$ & $\mathrm{Mg}^{2+}$ & SB & CTC & \multirow{2}{*}{$\begin{array}{l}\mathbf{V} \\
\%\end{array}$} & Areia & Silte & Argila \\
\hline $\mathrm{CaCl}_{2}$ & $\mathrm{~g} \mathrm{~kg}^{-1}$ & $\mathrm{mg} \mathrm{dm}{ }^{-3}$ & \multicolumn{6}{|c|}{$\mathrm{mmol}_{\mathrm{c}} \mathrm{dm}^{-3}$} & & \multicolumn{3}{|c|}{$\mathrm{g} \mathrm{kg}^{-1}$} \\
\hline 4,9 & 7 & 3 & 15 & 1,4 & 7 & 3 & 11,4 & 26,4 & 43 & 144 & 24 & 832 \\
\hline
\end{tabular}

M.O. - matéria orgânica; SB - Soma de bases $(\mathrm{K}+\mathrm{Ca}+\mathrm{Mg})$; CTC - capacidade de troca de cátions 
o volume total de água (L) por vaso aplicado durante a condução do experimento.

\section{RESULTADOS E DISCUSSÃO}

Pelos resumos das análises de variância (Tabela 2) temse que a interação salinidade do solo $\mathrm{x}$ níveis de umidade não exerceu efeitos significativos sobre o número de folhas emitidas, crescimento em altura e diâmetro da haste das plantas. Por outro lado, a salinidade do solo interferiu significativamente sobre as respectivas variáveis. Ao se considerar que a cebola é sensível aos efeitos dos sais (Ayers \& Westcot, 1999), constata-se maior expressividade da ação da salinidade do solo que dos níveis de umidade do solo, uma vez que apenas o número de folhas emitidas respondeu significativamente à ação da umidade do solo (Tabela 2).

Tabela 2. Resumo da análise de variância dos parâmetros, número de folhas por planta, altura da planta e diâmetro da haste

\begin{tabular}{lcccc}
\hline Causas da variação & GL & $\begin{array}{c}\text { N. folha } \\
\text { por planta }\end{array}$ & $\begin{array}{c}\text { Altura da planta } \\
\text { Salinidade (S) }\end{array}$ & $\begin{array}{c}\text { Diâmetro da } \\
\text { haste }\end{array}$ \\
Umidade (U) & 2 & $1,667^{* *}$ & $1952,2^{\star *}$ & $34,466^{\star *}$ \\
Interação (SxU) & 8 & $0,105^{*}$ & $14,5 \mathrm{~ns}$ & $0,460 \mathrm{~ns}$ \\
Resíduo & 30 & $0,048 \mathrm{~ns}$ & $49,98 \mathrm{~ns}$ & $0,914 \mathrm{~ns}$ \\
CV \% & & 7 & 25,08 & 1,0 \\
${ }^{* *},{ }^{*}$ - Significativo, respectivamente, a nível de 1 e 5 \% pelo teste F; ns - não significativo
\end{tabular}

O aumento da salinidade do solo reduziu linearmente o número de folhas das plantas (Figura 1A); este efeito foi mais drástico no tratamento de menor umidade do solo (25-50\% da CC) e maior nível salino (8 $\mathrm{dS} \mathrm{m}^{-1}$ ) promovendo declínio de $58 \%$ em relação ao tratamento de menor salinidade $\left(0,13 \mathrm{dS} \mathrm{m}^{-1}\right)$; esses resultados estão de acordo com os de Lacerda et al. (1998) ao constatarem que, com a redução de umidade do solo, os efeitos salinos se tornaram mais prejudiciais ao sorgo granífero e ao feijão guandu.

Infere-se que, de certa forma, há tendência de compensação de efeitos de umidade sobre a salinidade, ou seja, o número de folhas foi maior no nível de umidade mais elevado, o que pode ser atribuído a uma diluição na concentração salina do solo, pela maior quantidade de água, reduzindo os efeitos prejudiciais da salinidade.

Os diâmetros do pseudocaule no estágio final da cultura (Figura 1B), e a altura da planta (Figura 1D), também foram fortemente inibidos com o aumento do teor de sais na solução do solo.

O decréscimo no diâmetro do pseudocaule sob incremento de 2,0 dS m $\mathrm{m}^{-1}$ foi, respectivamente, de 43,5, 40,5 e 37\%, para as faixas de umidade entre 25-50, 50-75 e 75-100\% da capacidade de campo, indicando a interferência, de forma positiva, da umidade, no ajuste da planta ao aumento da concentração salina no solo. O comportamento dos dados está de acordo com Mangal \& Lal (1988) ao registrarem severa redução na altura e no número de folhas por planta e desenvolvimento de raízes em cebola, com o aumento do nível de salinidade do solo.

Com o resumo das análises de variância referentes ao diâmetro, comprimento e peso dos bulbos, verifica-se ação mais significativa da interação salinidade do solo $\mathrm{x}$ umidade do solo nas variáveis de produção (Tabela 3) que nas de crescimento da cultura (Tabela 2).

Tabela 3. Resumo das análises de variância dos diâmetros e peso dos bulbos de cebola

\begin{tabular}{lrccc}
\hline Causas da variação & GL & $\begin{array}{c}\text { Diâmetro do } \\
\text { bulbo }\end{array}$ & $\begin{array}{c}\text { Comprimento do } \\
\text { bulbo }\end{array}$ & Peso do bulbo \\
Salinidade (S) & 4 & $1554,17^{* *}$ & $2031,64^{* *}$ & $38861,4^{*}$ \\
Umidade (U) & 2 & $79,48^{* *}$ & $116,52^{*}$ & $716,7 \mathrm{~ns}$ \\
Interação (SxU) & 8 & $28,13^{*}$ & $79,00^{*}$ & $549,9 \mathrm{~ns}$ \\
Resíduo & 30 & 11,00 & 25,25 & 353,7 \\
CV \% & 12 & 13 & 27 & 27 \\
\hline
\end{tabular}

**, * - Significativo, respectivamente, a nível de 1 e 5 \% pelo teste $\mathrm{F}$; ns - não significativo

O aumento da salinidade na solução do solo também prejudicou o desenvolvimento dos bulbos da cebola (Figuras 1C e 1E). Pelas tendências dos dados do diâmetro transversal se percebem marcantes diferenças entre os tratamentos referentes à interação salinidade do solo x umidade do solo. Quanto à umidade do solo, nota-se que o aumento interferiu positivamente no diâmetro para os tratamentos mais próximos à capacidade de campo, uma vez que promove efeito diluidor dos sais (Cavalcante \& Cavalcante, 2006), situação em acordo com Galbiatti \& Castellane (1990) ao registrarem menor altura de planta, menor número de folhas por planta e também menor produtividade, quando foram repostos menos que $100 \%$ da evapotranspiração diária nas irrigações durante o ciclo da cultura de cebola.

$\mathrm{O}$ aumento do teor de sais no solo reduziu significativamente o comprimento do bulbo de cebola (Figura 1C). No tratamento com umidade na faixa de $25-50 \%$ CC, a redução foi de 81,85\%. Bernstein \& Ayers apud Mangal et al. (1989) detectaram redução de 50\% na produção de bulbos de cebola para condutividade elétrica do solo igual a $4,1 \mathrm{dS} \mathrm{m}^{-1}$ sugerindo, como limite de tolerância à salinidade, $3,47 \mathrm{dS} \mathrm{m}^{-1}$, para produção de bulbos de cebola.

Pela classificação dos bulbos com base no diâmetro transversal indicada na Tabela 4, notou-se que os tratamentos VIII

Tabela 4. Número de bulbos normais classificados segundo o diâmetro transversal

\begin{tabular}{clcccc}
\hline & $\begin{array}{c}\text { Tratamentos } \\
\text { Umidade e dS m }\end{array}$ & $\begin{array}{c}\mathbf{4 , 0}-\mathbf{5 , 5} \\
\mathbf{c m}\end{array}$ & $\begin{array}{c}\mathbf{2 , 5}-\mathbf{4 , 0} \\
\mathbf{c m}\end{array}$ & $\begin{array}{c}<\mathbf{2 , 5} \\
\mathbf{c m}\end{array}$ & Total \\
I & $(25-50 \%$ e CE $=0,13)$ & 6 & 3 & 0 & 9 \\
II & $(50-75 \%$ e CE $=0,13)$ & 8 & 1 & 0 & 9 \\
III & $(75-100 \%$ e CE $=0,13)$ & 8 & 1 & 0 & 9 \\
IV & $(25-50 \%$ e CE $=2,0)$ & 4 & 5 & 0 & 9 \\
V & $(50-75 \%$ e CE $=2,0)$ & 4 & 5 & 0 & 9 \\
VI & $(75-100 \%$ e CE $=2,0)$ & 3 & 6 & 0 & 9 \\
VII & $(25-50 \%$ e CE $=4,0)$ & 0 & 1 & 8 & 9 \\
VIII & $(50-75 \%$ e CE $=4,0)$ & 9 & 0 & 0 & 9 \\
IX & $(75-100 \%$ e CE $=4,0)$ & 9 & 0 & 0 & 9 \\
X* & $(25-50 \%$ e CE $=6,0)$ & 0 & 0 & 6 & 6 \\
XI* & $(50-75 \%$ e CE $=6,0)$ & 0 & 0 & 7 & 7 \\
XII* & $(75-100 \%$ e CE $=6,0)$ & 0 & 4 & 4 & 8 \\
XIII* & $(25-50 \%$ e CE $=8,0)$ & 0 & 0 & 4 & 4 \\
XIV* & $(50-75 \%$ e CE $=8,0)$ & 0 & 0 & 6 & 6 \\
XV & $(75-100 \%$ e CE $=8,0)$ & 0 & 0 & 9 & 9 \\
\hline
\end{tabular}

* Apresentaram charutos 
A.

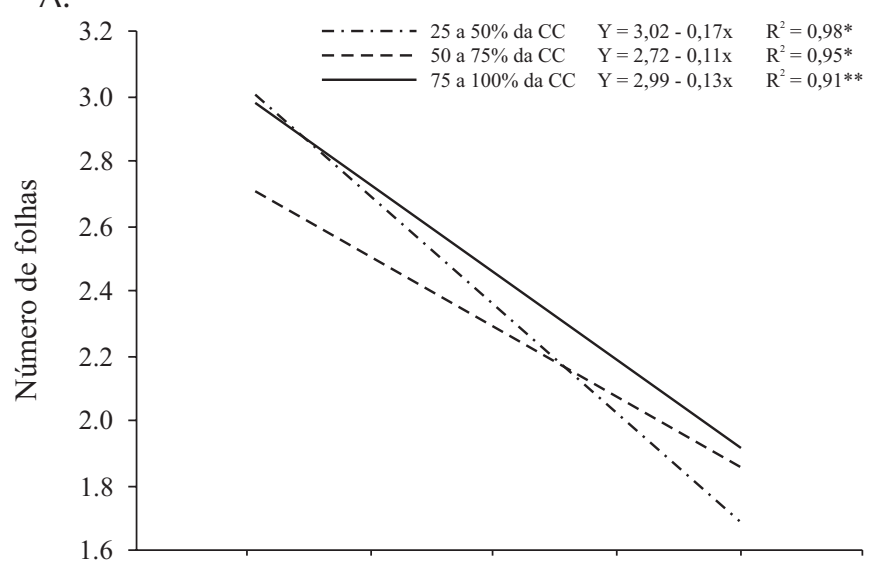

B.

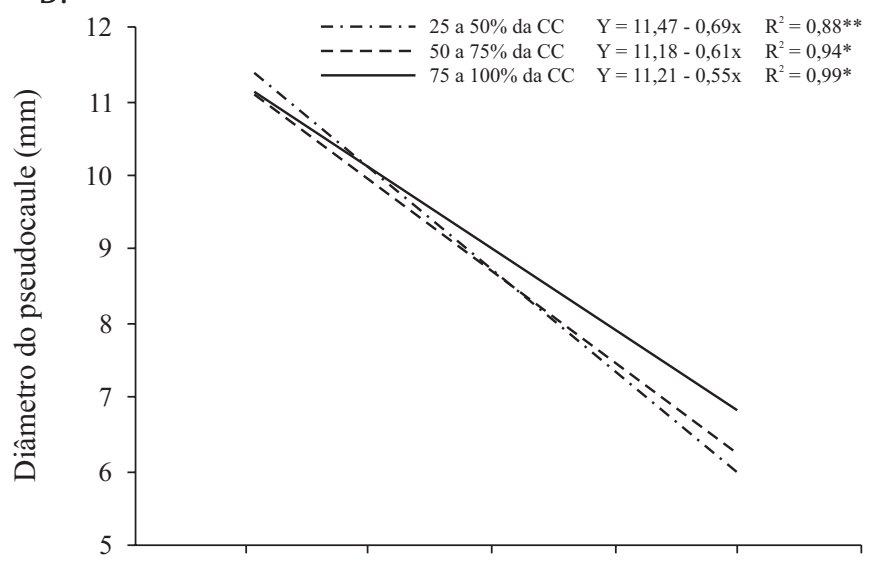

C.

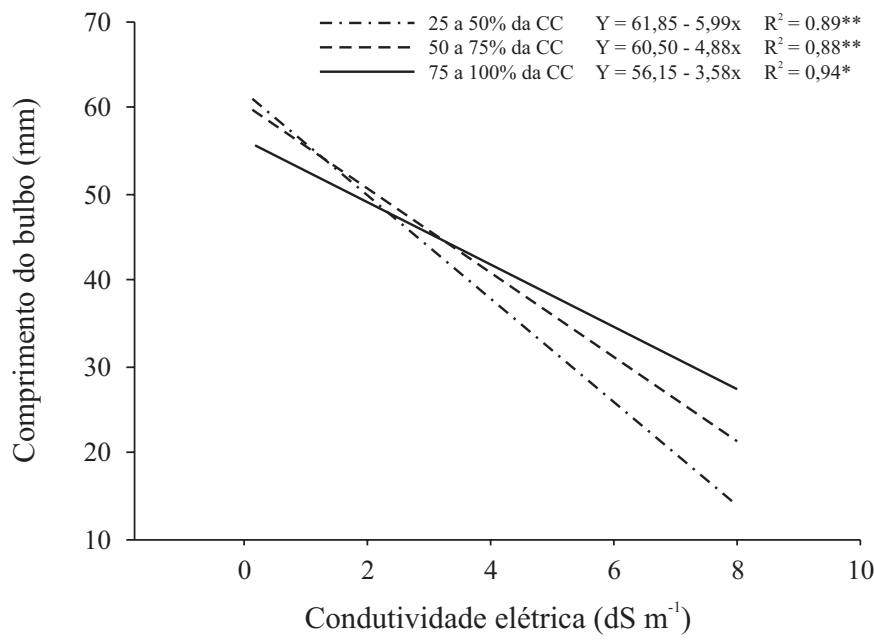

D.

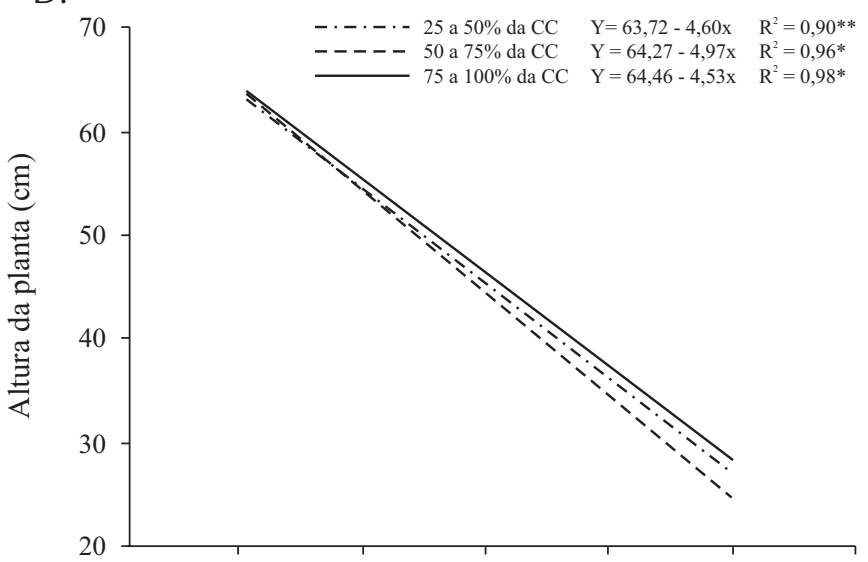

E.

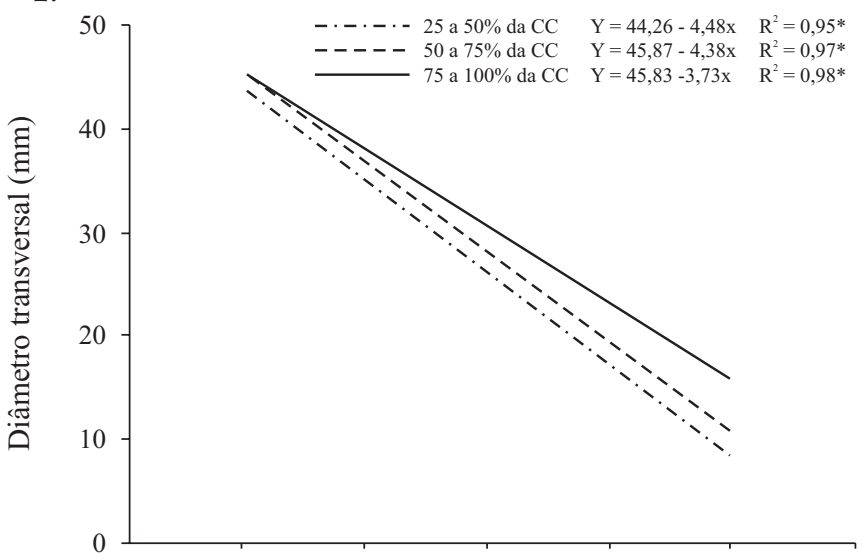

F.

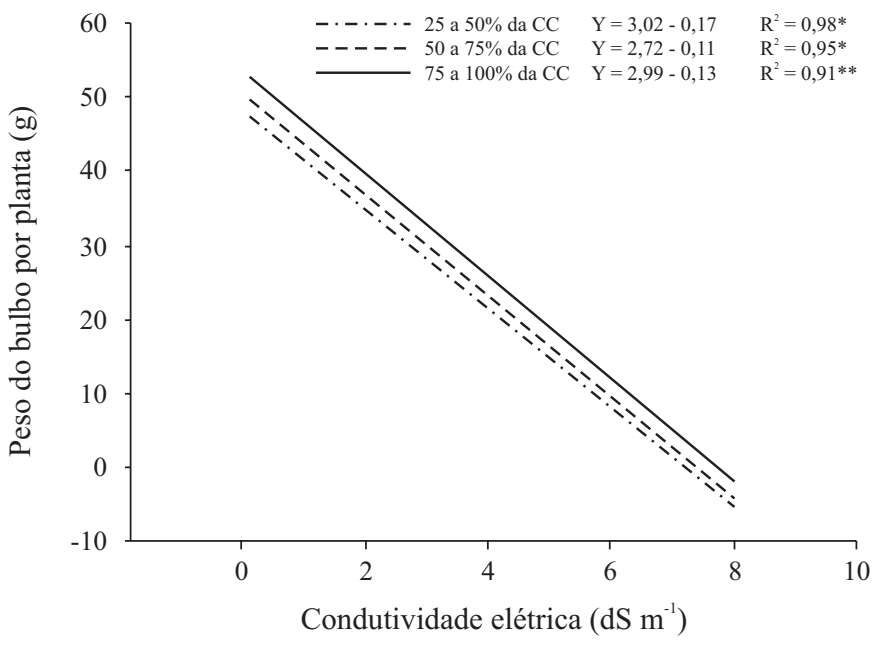

Figura 1. Número de folhas (A); diâmetro do pseudocaule (mm) (B); comprimento do bulbo (mm) (C); altura da planta (cm) (D); diâmetro do bulbo (mm) (E) e peso do bulbo (g) (F) em função da condutividade elétrica do extrato de saturação do solo e umidade do solo

e IX, correspondentes a $\mathrm{CE}=4,0 \mathrm{dS} \mathrm{m}^{-1}$ e $50-75$ e $75-100 \%$ CC, tiveram maior produção de bulbos com diâmetros entre 4,0 a 5,5 cm. Bulbos com diâmetros transversais de 4 a $8 \mathrm{~cm}$, formas arredondadas ou bojudas, têm a preferência dos consumidores, que adotaram referidas características como as de maior valor comercial (Gandim et al., 1994), e cujos resultados indicaram a $\mathrm{CE}=4,0 \mathrm{dS} \mathrm{m}^{-1}$ como limite à produção de bulbos de cebola em boas condições de umidade do solo.

A Figura 2 representa a eficiência de uso da água pelas plantas de cebola; observa-se que o volume de água utilizado pelas plantas diminuiu conforme aumentou a concentração de sais na solução do solo, indicando que o estresse salino induz ao menor consumo de água, em conseqüência da redução de crescimento (Rhoades \& Loveday, 1990). 


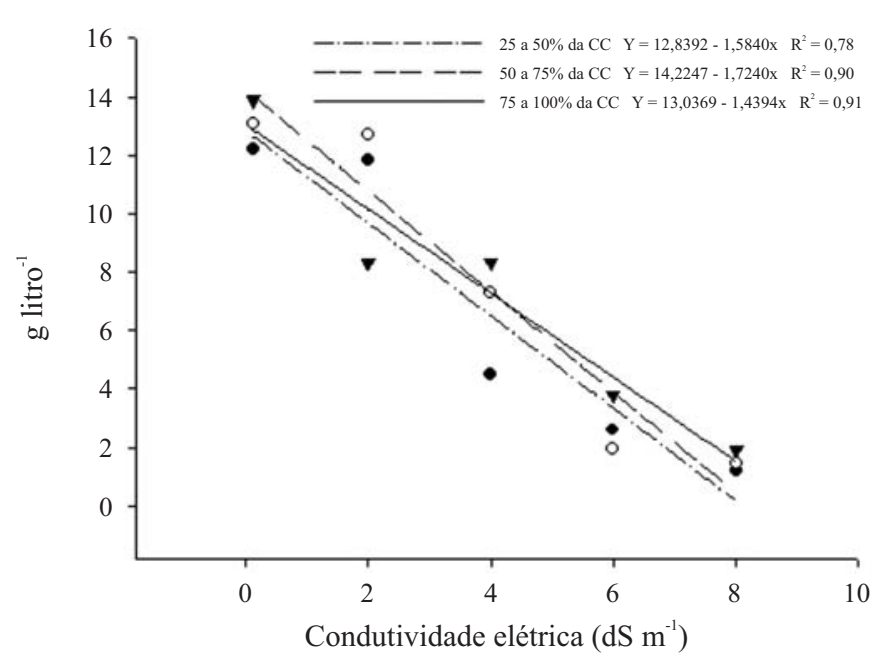

Figura 2. Eficiência de utilização de água $\left(\mathrm{g} \mathrm{L}^{-1}\right)$ por plantas de cebola em função da concentração de sais na parte aérea

\section{CONCLUSÕES}

1. O aumento da condutividade elétrica do extrato de saturação do solo inibiu o desenvolvimento vegetativo da cebola, reduziu o número de folhas por planta, o diâmetro do pseudocaule, a altura da planta, e o peso e o diâmetro dos bulbos, independente do nível de umidade do solo.

2. A salinidade do solo promoveu redução do consumo de água pelas plantas.

3. O limite de tolerância à salinidade para produção de bulbos de cebola foi de $4,0 \mathrm{dS} \mathrm{m}^{-1}$.

\section{LITERATURA CITADA}

Ayers, R. S.; Westcot, D. W. A qualidade da água na agricultura. 2.ed. Campina Grande: UFPB, 1999, 153p. Estudos FAO Irrigação e Drenagem, 29, revisado 1

Cavalcante, L. F.; Cavalcante, I. H. L. Uso da água salina na agricultura. In: Cavalcante, L.; Lima, E. M. (ed.). Algumas frutíferas tropicais e a salinidade. Jaboticabal: FUNEP. 2006. cap.I, p.1-9.

Dias, N. S.; Gheyi, H. R.; Duarte, S. N. Prevenção, manejo e recuperação dos solos afetados por sais. Piracicaba: ESALQ/USP, 2003. 118p. Série Didática,13

EMBRAPA - Empresa Brasileira de Pesquisa Agropecuária. Centro Nacional de Pesquisa de Solos. Sistema brasileiro de classificação de solos. Rio de Janeiro: Embrapa Solos, 1999. 412p.
Galbiatti, J. A.; Castellane, P. D. Efeito da irrigação e das adubações mineral e orgânica na cultivar de cebola piralopes, 1987. Horticultura Brasileira, v.8, n.1, p.24, 1990.

Gandim, C. L.; Guimarães, D. R.; Thomazelli, L. F. Caracterização de quatro cultivares de cebola lançadas em Santa Catarina, Brasil. Pesquisa Agropecuária Brasileira, v.29, n.12, p.1941-1945, 1994.

Gheyi, H. R.; Correia, K. G. Fernandes, P. D. Salinidade do solo e crescimento e desenvolvimento das plantas. In: Nogueira, R. J. M. C.; Araújo, E. de L.; Willadino, L. G.; Cavalcante, U. M. T. (ed.). Estresses ambientais: Danos e benefícios em plantas. Recife: UFPE, 2005, p.138-154.

Lacerda, C. F. Interação salinidade x nutrição mineral. In: Nogueira, R. J. M. C.; Araújo, E. de L.; Willadino, L. G.; Cavalcante, U. M. T. (ed.). Estresses ambientais: Danos e benefícios em plantas. Recife: UFPE, 2005, p.127-137.

Lacerda, D. A.; Cavalcante, L. F.; Feitosa Filho, J. C.; Vieira, J. E.; Cavalcante, I. H. L.; Santos, C. J. O. Dispersão de argila e acúmulo de sais em dois solos não salinos irrigados com água salgada. Anais do Curso de Pós-Graduação em Manejo de Solo e Água, v.20, p.11-22, 1998.

Lima, L. A. Efeito de sais no solo e na planta. In: Gheyi, H. R.; Queiroz, J. E.; Medeiros, J. M. (ed.). Manejo e controle da salinidade da agricultura. Campina Grande: UFPB; SBEA, 1997. p.113 -136.

Mangal, J. L.; Lal, S. Salt tolerance behaviour of Kharif onion variety N.53. Haryana Journal Horticultural Science, v.17, n.1-2, p.78-82, 1988.

Mangal, J. L.; Lal, S.; Hooda, P. S. Salt tolerance of onion seed crop. Journal Horticultural Science, v.64, n.4, p.475-477, 1989.

Mascarenhas, M. H. T. Cultivares de cebola. Informe Agropecuário, v.6, n.62, p.17-20, 1980.

Millar, A. A. Manejo racional da irrigação: uso de informações básicas sobre diferentes culturas. Brasília: Instituto Interamericano de Cooperação para a Agricultura, 1984. 56p.

Rhoades, J. D.; Loveday, J. Salinity in irrigated agriculture. In: Stewart, D. R.; Nielsen, D. R. (ed) Irrigation of agricultural crops. Madison: ASA, CSSA, SSSA, 1990. p.1089-1142. Agronomy, 30

Sousa, G. B. Interação biofertilizante x salinidade x volume de substrato na germinação e crescimento inicial do maracujazeiro-amarelo. Areia: UFPB, 2006. 78p. Dissertação Mestrado

Wannamaker, M. J.; Pike, L. M. Onion responses to various salinity levels. Journal of American Society Horticultural Science, v.112, n.1, p.49-52, 1987.

Ziska, L. H.; Seemann, J. R.; de Jong, T. M. Salinity induced limitations on photosynthesis in Prunus salicina, a deciduous tree species. Plant Physiology, v.93, p.864-70, 1990. 